\title{
Status of Current Clinical Trials in Diabetic Polyneuropathy
}

\author{
Vera Bril
}

\begin{abstract}
Peripheral polyneuropathy is the most frequent complication of diabetic mellitus. In spite of many clinical trials of different specific interventions for diabetic polyneuropathy, intensive glycemic control remains the only effective specific therapy currently available for this troublesome complication. This systematic overview reports the status of current clinical trials in diabetic polyneuropathy with an emphasis on those interventions directed towards specific pathophysiological derangements. A discussion of clinical trials of agents directed towards relieving painful symptoms of diabetic polyneuropathy concludes this overview.
\end{abstract}

RÉSUMÉ: Revue des essais cliniques en cours dans la polyneuropathie diabétique. La polyneuropathie périphérique est la complication la plus fréquente du diabète. En dépit de nombreux essais cliniques, le contrôle glycémique intensif demeure le seul traitement spécifique efficace disponible actuellement pour traiter cette complication importante. Cette revue systématique fait le point sur les essais cliniques en cours ciblant des perturbations physiopathologiques spécifiques. Une discussion des essais cliniques d'agents utilisés pour soulager la douleur due à la polyneuropathie diabétique est présentée en guise de conclusion à cette revue.

Can. J. Neurol. Sci. 2001; 28: 191-198

Diabetic polyneuropathy (DPN) remains one of the most challenging therapeutic areas for physicians, particularly neurologists, endocrinologists and primary care physicians. After years of intensive investigation, answers to some basic questions remain elusive; for example, what causes some patients to perceive unremitting pain and others to remain virtually asymptomatic despite similar durations of diabetes, levels of glycemic control and degrees of nerve fibre loss on pathological review. Specific interventions directed at the neuropathy are few: only intensive glycemic control helps reduce prevalence of neuropathy. ${ }^{1-4}$ The benefits for patients with Type 2 diabetes are far less certain. ${ }^{3}$ Multiple agents have failed phase 3 trials for various reasons and are unavailable for use in DPN.

This review focuses on the clinical trials of new treatments for DPN providing an update on the current status of investigation into this disorder. A discussion of symptomatic therapy for painful symptoms of diabetic polyneuropathy concludes this review.

\section{Clinicaltrials fOR DPN: \\ SPECIFIC ANTI-NEUROPATHY INTERVENTIONS}

Specific interventions directed towards DPN are based on presumed pathophysiological mechanisms which have been recently reviewed. ${ }^{5,6}$ Disordered nerve function in diabetes can be considered in several broad categories: metabolic, vascular, genetic, protein glycosylation, and neurotrophism. These are not mutually exclusive but tend to overlap and interact. Many nerve derangements demonstrated in animal models have not been shown in human subjects. ${ }^{7}$ The fundamental abnormality, other than hyperglycemia, leading to DPN in man is uncertain. For further details concerning pathophysiology of DPN, the reader is referred to recent reviews. ${ }^{5,8,9-15}$ Table 1 lists the specific interventions discussed in this paper.

\section{Hyperglycemia}

The evidence for the value of reducing hyperglycemia is strong in Type 1 diabetes. $^{2}$ The Diabetes Control and Complications Trial proved conclusively that intensive glycemic control reduces the prevalence of confirmed DPN in young men by $64 \%$ as measured by clinical features, nerve conduction studies (NCS) and autonomic system testing. ${ }^{1,2,16}$ The effect was noted after five years of intensive intervention. Trials of intensive control in smaller numbers of patients treated for briefer durations, such as one year, have failed to demonstrate any alteration in symptoms, signs, NCS, vibration perception thresholds (VPT) and thermal thresholds. ${ }^{17}$ Other trials have shown electrophysiological improvements after shorter periods

\footnotetext{
From the Toronto General Hospital, UHN, University of Toronto, Toronto, Ontario, Canada.

Received February 13, 2001. Acceptedin finalform May 29, 2001. Reprint requests to: Vera Bril, Toronto General Hospital, UHN, University of Toronto, EN11-209, TGH, 200 Elizabeth St, Toronto, Ontario, M5G 2C4 Canada
} 
of intensive control. ${ }^{18}$ Presumably, the latter implies eventual clinical benefits if the positive therapeutic intervention is maintained. The UK Prospective Diabetes Study proves that better control for many years can improve neuropathic indices although limited end-points were performed in this study. ${ }^{3}$ After 15 years of intervention, no difference in ankle and knee reflexes, or erectile dysfunction was observed between groups, but vibration perception threshold was lower after nine years of therapy in the better controlled group. The relative risk of neuropathy as measured with the biothesiometer was $0.60(95 \%$ confidence intervals: $0.39-0.90, \mathrm{p}=0.0052)$ with better control. Navarro's study showed that pancreatic transplant can help prevent progression of clinical, autonomic and electrophysiological parameters in both men and women. ${ }^{19}$ This cumulative evidence along with epidemiological studies, such as that by Pirart, ${ }^{20}$ which show more neuropathy in those with worse control after 25 years of diabetes, have changed practice patterns and recommendations for treatment in North America. Recent guidelines recommend that any patient with $\mathrm{HbA1C}>$ $8.4 \%$ should be subjected to "additional action", and those with HbA1C between 7.1 and $8.4 \%$ have suboptimal control which may require action. ${ }^{21,22}$

\section{Aldose reductase inhibitors}

The aim of aldose reductase inhibitor (ARI) therapy is to protect the nerve from the effects of overactivity of the polyol pathway caused by chronic hyperglycemia (Figure). In states of uncontrolled hyperglycemia, activation of this pathway results in accumulations of sorbitol and fructose and concomitant decreases in Na-K-ATPase. These changes are prevented by ARI administration in animal models and man. ${ }^{23,24}$ Unfortunately, none of the agents which were promising in the laboratory and

Table : 1 Specific Interventions Investigated for the Treatment of Diabetic Polyneuropathy

\begin{tabular}{lll}
\hline Target & Intervention & Specific Agents \\
Hyperglycemia & Intensive glycemic & Insulin \\
control & & pancreatic/islet cell \\
transplant & oral hypoglycemic \\
& & agents+ insulin \\
Polyol pathway & Aldose reductase & alredase \\
& inhibitors & sorbinil \\
& & statil \\
& & FK-366/zenarestat \\
& & tolrestat \\
Non-specific metabolic & Ubiquitous & zopolrestat \\
Neurones & Neurotrophins & epalrestat \\
& & alcar \\
& & NGF \\
Vascular & Anti-oxidants & $\alpha$-lipoic acid \\
& & $\gamma$-Linolenic acid \\
& & PKCi \\
& &
\end{tabular}

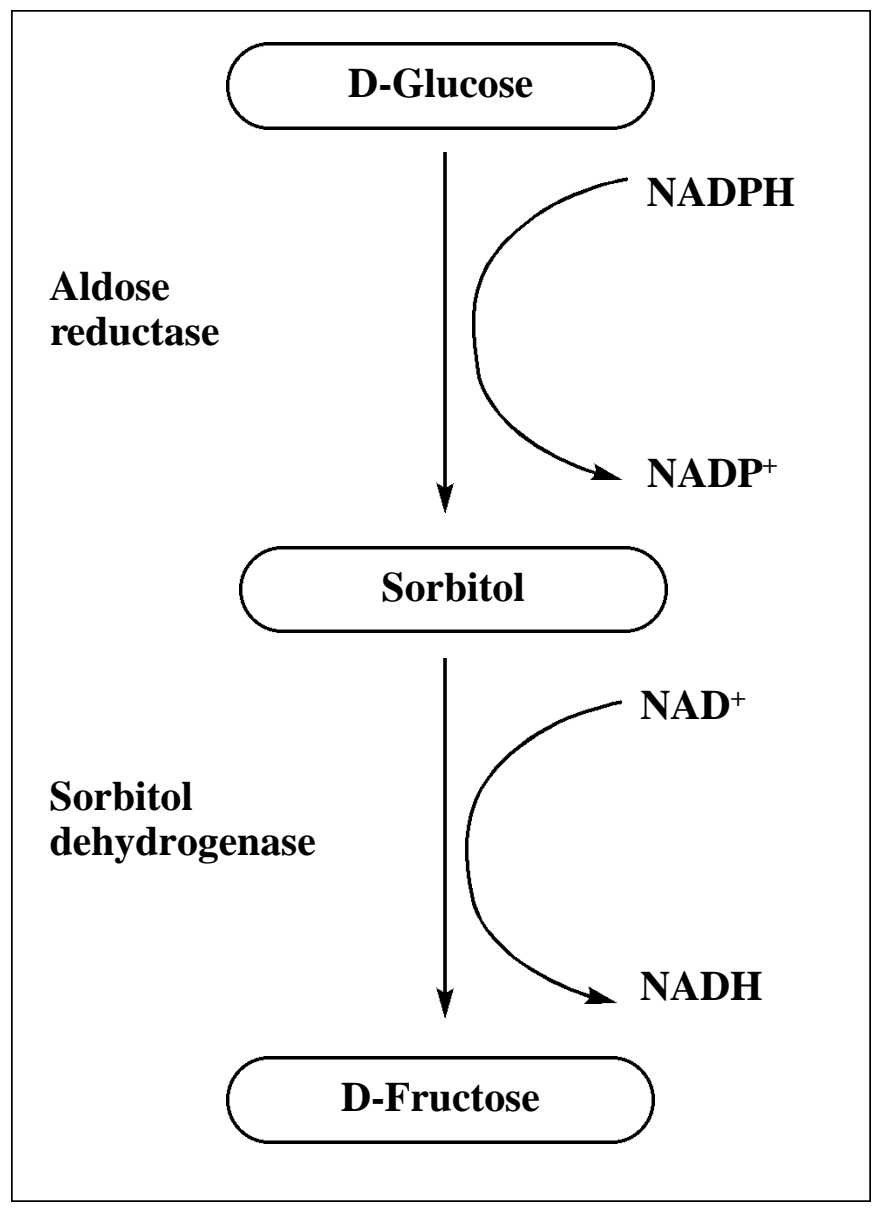

Figure: Polyol Pathway

early clinical trials proved effective in phase III trials for various reasons reviewed below. Currently, no aldose reductase inhibitor agent has proven effective for the therapy of DPN.

Sorbinil, the first agent extensively studied, was associated with toxic epidermal necrolysis as a complication in two patients. The methodology for measurement of the efficacy parameters in the pivotal neuropathy study was imperfectly defined and the results not monitored closely or expertly. The treatment interval lasted only 12 months. A single centre reporting results of pre- and post-treatment sural nerve biopsy parameters showed efficacy, ${ }^{7}$ but morphology was not a pivotal parameter in the overall study. The primary efficacy parameters from all study centres combined were negative. In another trial directed at retinopathy, neuropathy measures did not improve with sorbinil therapy. ${ }^{25}$ However, the same problem with data quality control existed in that trial.

Statil, a much safer ARI, was used for 18 months in a doubleblind, placebo-controlled fashion. The methodology for measuring the efficacy end-points of NCS and quantitative sensory thresholds was carefully defined and the results monitored closely. The quality of the data was excellent. Unfortunately, the results were negative. ${ }^{26}$ No difference in nerve 
conduction or quantitative sensory threshold parameters was found between groups treated with drug or placebo. After these disappointing results were found, further investigation revealed that statil did not penetrate human nerve. This compound demonstrated species differences in aldose reductase inhibitor activity as rat nerve showed the expected benefits with this intervention. ${ }^{27,28}$ Another study of statil therapy in diabetic autonomic neuropathy showed similarly disappointing results. ${ }^{29}$

Tolrestat proved problematic in that some patients developed hepatic side-effects in an idiosyncratic manner on this medication. Severe elevations in hepatic enzymes were observed in a few patients. Furthermore, the interventions were limited to 18 months in the therapeutic trials; a very short time to show significant change in parameters of DPN. Small changes in motor nerve conduction velocities were observed but their meaning was questioned. Positive changes in nerve biochemistry and sural nerve morphology were observed in subjects treated with tolrestat in long-term, uncontrolled studies, ${ }^{30}$ but sural nerve parameters did not show sufficient change during the pivotal 12 month trials to establish therapeutic efficacy. Different research definitions of DPN emerged ${ }^{31}$ and modified requirements to prove therapeutic value. Longer study intervals may well have demonstrated true therapeutic benefits with this compound but development was halted due to the potential hepatic toxicity. Also, the degree of inhibition of the aldose reductase enzyme by tolrestat may not have reached levels now considered necessary to have the necessary effects in animal and human nerves. ${ }^{32}$

FK-366 showed great promise in a phase 2 study in which both morphometry and electrophysiology showed benefits after 12 months of ARI intervention. ${ }^{33}$ Large-scale phase 3 trials of two years of treatment in the pivotal North American study (assessing endpoints of NCS and VPT) and five years of treatment in the foot ulcer prevention trial were terminated only recently in late 2000 due to unexpected dose-dependent elevations in creatinine levels in those on zenarestat. Clearly an agent having the potential to cause nephrotoxicity cannot be used to treat the complication of DPN since these patients are prone to develop nephropathy related to their diabetes and DPN will require chronic therapy. This drug showed $85-90 \%$ aldose reductase inhibition and a positive effect on the electrophysiological parameters at the time of termination, similar to those changes previously seen in the phase 2 study.

Zopolrestat is another ARI whose development was halted in 1999. The interim analysis of sural nerve fiber density after 18 months of treatment in a planned three year intervention program failed to demonstrate sufficient efficacy in this morphological parameter, one of two primary efficacy end-points. Although electrophysiology was also being measured, a single parameter is insufficient to demonstrate efficacy in any pivotal study in DPN. Although the phase 2 study was successful, the dose used was twice that used in the phase 3 study and likely accounts for the different results observed. One may speculate that the level of inhibition of nerve aldose reductase was weaker than expected with the lower dose, and hence insufficient to produce the hopedfor results in the phase 3 studies. ${ }^{32}$

Epalrestat, another ARI, is available in Japan. ${ }^{34,35}$ However, the studies have not established sufficient efficacy for use in
North America. The degree of aldose reductase inhibition may not be as high as currently considered efficacious.

More potent ARIs (fidarestat, IDD-676, AS-3201) are being investigated for efficacy. A phase 2 trial of fidarestat is underway and phase 2 trials of IDD-676 and AS-3201 should start later this year in North America. Whether these agents will prove sufficiently safe and efficacious for long-term treatment of DPN in human subjects remains to be determined.

\section{OTHER METABOLIC INTERVENTIONS}

Another therapeutic intervention which failed was acetyl-1carnitine, Alcar. Based on results from animal studies, this drug had multiple potential benefits for human nerves including: effects on free fatty acid metabolism, nitric oxide synthase activity, mitochondrial function, membrane phospholipid structure, axonal transport, etc. ${ }^{36-39}$ Preliminary trials were positive. Then an excellent 12 month study which measured NCS and sural nerve morphology from pre- and post-treatment biopsies $^{40}$ was carried out. Unfortunately, no effects on human nerve function and/or structure were observed after 12 months of therapy and further development was halted.

\section{Neurotrophins}

The roles of neurotrophins in neurological disorders are widespread. ${ }^{41}$ Those directed at peripheral nerve function are promising therapeutic targets for DPN. ${ }^{42}$ Nerve growth factor is particularly attractive, due to its name which implies the ability to stimulate nerves to grow. This agent has selective effects for sensory and autonomic neurons. ${ }^{43,44} \mathrm{~A}$ phase 2 trial proved surprisingly efficacious in parameters subserved by smaller fibers (pain perception and thermal thresholds) after six months of therapy ${ }^{44,45}$ but the phase 3 study was a resounding failure with the same efficacy parameters and 12 months of therapy. ${ }^{46}$ This is most likely due to the fact that the phase 2 trial was effectively unblinded as, in that trial, the NGF treatment consisted of painful injections compared to injections of a painless placebo. Approximately $90 \%$ of the patients and physicians guessed the treatment arm correctly. In the phase 3 trials, the placebo was designed to be uncomfortable, and the observers for the primary end-points (Neuropathy Impairment Score in the lower limbs [NIS-LL] $^{47,48}$ and quantitative thermal thresholds) were kept blinded as to the treatment group for each subject but also all adverse events reported by the patients. Lack of efficacy in the US pivotal trial resulted in termination of the research program worldwide in 1999, including a study on the prevention of foot ulcers with three years of double-blind treatment.

Neurotrophin 3 (NT3) was another promising neurotrophin with selective effects on large nerve fibers. Recently a small six month phase 2 trial was completed but changes in VPT were not observed and further development has been halted, although NT3 did not produce any undue toxicity. Further development is not currently underway (personal communication).

Prosaposin is another neurotrophin occurring naturally in human milk and CSF. A synthetic form, Prosaptide ${ }^{\mathrm{TM}} \mathrm{TX} 14$ (A) has shown efficacy in animal models ${ }^{49,50}$ and symptomatic improvements in painful DPN in a phase 2 study (personal communication). Further clinical trials are necessary to confirm the efficacy of this intervention, both for painful symptoms and also for nerve function. 


\section{VASCULAR INTERVENTIONS}

\section{Anti-oxidant therapy}

Alpha-lipoic acid (thioctic acid) has proved efficacious in phase 2 studies including small numbers of patients. This agent is a potent lipophilic free radical scavenger acting as an antioxidant and improving blood flow. ${ }^{51}$ It alleviates oxidative stress in diabetic nerve by multiple potential mechanisms as outlined in the review by Ziegler. A three-week intervention trial with intravenous therapy had questionable validity due to the high number of protocol violators withdrawn from study analysis. The results of this trial indicated more benefit with thioctic acid than one would expect even with intensified glycemic control. ${ }^{17}$ After one year of intensive treatment of hyperglycemia, no improvements in clinical features, NCS, or quantitative sensory thresholds were observed. ${ }^{17}$ These results cause some concern about the dramatic changes reported in the ALADIN study of thioctic acid administered by intravenous infusion daily for three weeks. ${ }^{51,52}$ However, the results led to further development including a phase 3 trial of oral treatment (NATHAN Study) in North America and Europe. This four year intervention trial, designed to show slowing of progression of DPN as measured by clinical, electrophysiological and autonomic measures of neuropathy, is underway. ${ }^{51}$ The results of another three month intervention trial with intravenous thioctic acid are not yet available.

\section{Blood flow}

The protein kinase $\mathrm{C}$ (PKC) pathway is activated by hyperglycemia resulting in reduced blood flow. ${ }^{53,54}$ Inhibition of this pathway may normalize endoneurial blood flow and improve neuropathy. Currently, a 12-month phase 2 study utilizing efficacy parameters of NIS-LL, NCS, and quantitative sensory thresholds is nearing completion. Further development will naturally depend on the outcome of the phase 2 study.

Other vascular interventions have been somewhat disappointing; such as pentoxyphylline, a smooth muscle relaxant, and sabeluzole, a calcium channel blocker, which have proven negative in phase 2 trials. ${ }^{55-57}$ Gamma-linolenic acid plays a role in phospholipid structure and microcirculation. $\omega-6$ essential fatty acid metabolism is altered with abnormal prostanoid synthesis which contributes to impaired nerve perfusion. These findings have led to clinical trials of $\gamma$-linolenic acid (GLA)-containing oils. ${ }^{13}$ A phase 2 study of 111 patients following NCS and clinical signs showed large deteriorations in placebo patients and improvements in drug-treated subjects; ${ }^{58,59}$ again unexpected given the lack of NCS changes in placebo subjects in one year observed in other trials. ${ }^{60}$ The study may have been somewhat unbalanced with respect to distribution of patients across sites perhaps contributing to the results reported.

\section{Others}

Other prospective neuropathy treatments include stimulation of neurite outgrowth, activation of protective receptors such as with immunophilin ligand treatment and inhibition of abnormal excitatory activity. These interventions will need to be designed specifically for DPN to avoid bystander neurotoxicity with chronic use. None of these interventions is yet ready for phase 3 trials.

\section{Summary of current status of specific therapies for DPN}

To date, no specific intervention has proven effective for DPN other than intensive glycemic control. Even with major efforts to achieve optimal glycemic control, neuropathy is unlikely to be reversed. Furthermore, the prevalence of DPN does not drop to zero, as intensive control does not equate with euglycemia in more than $15 \%$ of highly motivated young males. ${ }^{1}$ The associated cost is an increase in the incidence of serious hypoglycemic episodes. Several major trials of specific antineuropathy drugs have failed within the last two years: NGF and zopolrestat due to lack of sufficient efficacy, zenarestat due to unacceptable toxicity. However, newer, more potent ARIs with different toxicity profiles may prove efficacious and sufficiently safe for clinical use. Vascular interventions have been disappointing to date but others such as PKC inhibitors and thioctic acid are being investigated actively. Still, the only valid advice to offer patients currently is to intensify their glycemic control indefinitely to prevent progression. Reversal of neuropathy may be an unrealistic goal.

\section{Clinical tRIALS FOR DPN: SYMPTOMATIC INTERVENTIONS}

Many patients with DPN have pain at some time during their neuropathy course. At least $10 \%$ of patients with DPN are affected at any one time. ${ }^{61}$ The prevalence of painful symptoms in DPN is likely even higher as $34 \%$ of patients classified as having DPN, in a Toronto cohort of diabetic patients being screened for neuropathy, ${ }^{62}$ reported painful symptoms referable to their neuropathy. A US National Health Interview Survey, published in 1965 , reported that $22.5 \%$ of patients with diabetes experienced leg pain in the preceding month although it is unclear how many of these subjects had DPN. ${ }^{63}$ Thus pain in the lower extremities is a frequent complaint in those with DPN.

These painful symptoms are highly variable in nature, and often debilitating and difficult to control but are amenable to therapy. The painful symptoms are variably described as burning, freezing, tingling, aching, cramping, shooting and electric shock-like. Some authorities advocate different symptomatic interventions based on the nature of the painful complaint. ${ }^{64}$ Many patients have a mixture of all types of these painful symptoms and respond in a non-specific manner to interventions.

Many symptomatic interventions are available as shown in Table 2. ${ }^{65}$ These are categorized as: analgesics and adjuvant analgesics, the latter drugs developed for other indications primarily but having analgesic potency. The major groups with demonstrated efficacy for DPN are: opioids, anticonvulsants, antiarrhythmics, and antidepressants. Many good clinical trials are available showing efficacy of these interventions in patients with painful DPN. ${ }^{66-79}$ The trials are similar in that the response rate is incomplete and only about $60-70 \%$ of carefully selected patients achieve at least a moderate $(\geq 50 \%)$ reduction in pain. The response rate in the clinic is predictably lower. Thus expectations need to be realistic in that pain is rarely relieved completely and not every patient responds to intervention.

The therapeutic approach is similar with all symptomatic agents. ${ }^{80}$ The dose is started at a low level, and slowly escalated to avoid undue toxicity. The interval between dose escalations 
varies in each clinic but one week is a reasonable period of time. Upward titration is continued until analgesia is effective, intolerable side-effects occur, or the maximum conventional dose is reached. ${ }^{80}$ Great variability in responses between patients and within a single patient is to be expected. Side-effects are unpredictable within a class and between classes. A "trial-anderror" paradigm is employed and patients need to be aware of this approach. Typically, sequential drug monotherapy is necessary, and combination therapy may prove effective when single agents fail to provide analgesic benefit.

\section{Anti-depressants}

The first choice of adjuvant analgesic in many patients is one of the tricyclic antidepressants (TCA). These agents act by blocking uptake in norepinephrinergic pathways and increasing net inhibitory function. ${ }^{67}$ Their effect is independent of their antidepressant activity. ${ }^{70}$ The efficacy of TCA has been demonstrated in multiple trials. ${ }^{70-72,75,81,82}$ All of the TCA are helpful including amitriptyline, doxepine, imipramine, desipramine, nortriptyline and clomipramine. All have significant side-effects including orthostatic hypotension, arrhythmia, increased risk of hip fracture in the elderly, somnolence, weight gain, dry mouth, blurry vision, precipitation of acute angle-closure glaucoma, constipation, obstipation, urinary retention and delirium. Their analgesic potency is comparable and the choice of agent often depends on the clinical situation. Failure to respond to one agent should be the signal to try another within the class. Amitriptyline is frequently the first choice of TCA. The suggested starting dose is $10 \mathrm{mg}$ at bedtime, which can be titrated upwards slowly until the patient achieves analgesia, or intolerable side-effects (often about 50-60 mg daily, although some patients achieve doses of 100-150 mg daily).

All antidepressant drugs are not equally effective in the

Table 2: Symptomatic Therapies for Painful Symptoms of Diabetic Polyneuropathy

\begin{tabular}{|c|c|c|}
\hline Category & Class & Specific Agent \\
\hline \multirow[t]{3}{*}{ Analgesics } & $\begin{array}{l}\text { Non-steroidal anti- } \\
\text { inflammatory agents, } \\
\text { Acetaminophen }\end{array}$ & First line \\
\hline & Opioids & tramadol \\
\hline & & oxycodone \\
\hline \multirow[t]{8}{*}{ Adjuvant Analgesics } & Anti-depressants & amitriptyline \\
\hline & & desipramine \\
\hline & & imipramine \\
\hline & & nortriptline \\
\hline & & doxepine \\
\hline & Anti-arrhythmics & mexiletine \\
\hline & Anti-convulsants & carbamazepine \\
\hline & & gabapentin \\
\hline \multirow[t]{2}{*}{ Topical agents } & Substance Pdepletor & capsaicin \\
\hline & Local anaesthetics & lidocaine \\
\hline \multirow[t]{2}{*}{ Specific therapies } & Vasodilators & $\alpha$-lipoic acid \\
\hline & Neurotrophins & Prosaptide ${ }^{\mathrm{TM}} \mathrm{TX} 14(\mathrm{~A})$ \\
\hline
\end{tabular}

treatment of painful DPN. Trazadone and fluoxetine have proven negative in controlled trials although inconsistent results have been reported. ${ }^{71}$

Recently, venlafaxine has been reported to be efficacious for the treatment of DPN, ${ }^{83,84}$ providing another therapeutic option although controlled trials need to be done. The starting dose recommended is $37.5 \mathrm{mg}$ daily and increasing to $75 \mathrm{mg}$ daily. Novel antidepressants are being tested in controlled clinical trials for this therapeutic intervention.

\section{Anticonvulsants}

The effective drugs in this category are carbamazepine and gabapentin. ${ }^{66,73,75,78,85,86}$ Carbamazepine may act by binding voltage-dependent sodium channels. By this action, neurons are able to fire at moderate rates but not more rapidly as might be observed in the ictus. Typically, a low starting dose of $100 \mathrm{mg}$ daily and slow upward titration to a dose of 800-1000 mg daily in divided doses are suggested. The side-effects of carbamazepine are limiting and include: headache, drowsiness, ataxia, diplopia, agranulocytosis, hepatotoxicity and Stevens-Johnson Syndrome. Many patients do not tolerate sufficient drug to achieve analgesia.

Gabapentin is a novel anticonvulsant which has been shown in a recent study to have analgesic potency for DPN. ${ }^{78,86}$ The mechanism of action is unknown. It is much better tolerated than carbamazepine or the TCA and often is the drug of first choice for the therapy of painful DPN. Not all formularies allow use of gabapentin for this indication thus barring some patients from the benefits of this therapy. The starting dose of gabapentin is $400 \mathrm{mg}$ once daily, with a slow titration up to $3600 \mathrm{mg}$ daily in divided doses. The common side-effects are drowsiness, ataxia, anergy, facial swelling and dizziness. As typical of other adjuvant analgesics, gabapentin relieved pain in approximately $60 \%$ of patients by at least $50 \%$. Strikingly, gabapentin also showed efficacy in multiple secondary end-points measured in the randomized, controlled clinical trial including: reducing sleep interference, improving mood, and improving the patient's overall sense of well-being.

Other than carbamazepine and gabapentin, the proof for antiepileptic drugs is limited and none of the other anticonvulsants are suggested for treating painful DPN at this time. Topiramate, another novel anticonvulsant agent, was effective in a preliminary study which was, however, questionably blinded due to mental status changes in the drug group as compared to the placebo group. The results were promising enough to lead to a further, large scale, phase $2 / 3$ trial currently underway in the United States. Adequate therapeutic trials in DPN are not available for any of the other newer anti-epileptic drugs. Pregabalin, which has been shown effective for this indication in a controlled clinical trial, is not yet available.

\section{Other adjuvant analgesics}

Antiarrhythmics can be used but are problematic given that many of these patients have coronary artery disease and the potential for cardiac arrthymias which induces reluctance to use mexiletine. Mexiletine, an antiarrhythmic with local anaesthetic properties and similar to lidocaine in structure and acitivity, blocks voltage-gated sodium channels but the mechanism of action in DPN is unknown. Studies have shown efficacy in relieving painful symptoms in DPN. ${ }^{87-89}$

Topical therapy with local anaesthetics or capsaicin are also 
options for this challenging disorder. ${ }^{90,91}$ Capsaicin, a derivative of hot, red peppers depletes substance P from nerve terminals, and may provide relief in selected patients.

\section{Opioids}

Finally, some patients benefit from opioid therapy, although chronic intervention with this form of treatment is problematic and many patients fail to respond although remaining on chronic therapy. However, intervention with an appropriate opioid may provide relief without significant adverse effects. ${ }^{68,69}$ Tramadol has proven effective for painful DPN both in short-term and longer studies. ${ }^{68,92}$ Oxycodone is being investigated currently in a double-blind, placebo-controlled trial for this indication. The side-effects are those typical of opiates: nausea, constipation, headaches and somnolence.

\section{SUMMARY OF CURRENTSTATUS OFSYMPTOMATIC THERAPIESFOR PAINFUL SYMPTOMS OF DPN}

In summary, many symptomatic interventions are available to the clinician for intervention in DPN. None are universally effective and all are associated with significant side-effects which must be weighed against potential benefit when prescribed for patients. Few of these agents have undergone direct comparison for efficacy in clinical trials, although statistical manipulation allows an estimate of relative efficacy of different symptomatic interventions. ${ }^{93}$ Novel agents are being investigated. In some patients the pain of DPN subsides after several years as the neuropathy progresses. However, many other patients complain of unremitting, severe pain, and others simply adjust to their symptomatology. The reassurance that symptoms subside as nerves die is hardly rewarding to the physician. In addition, caution is advised when discussing the benefits of improved glycemic control with these patients. Painful symptoms typically develop after about 10 years of hyperglycemica, and improved control will likely need to be maintained for many years prior to producing any significant benefit for patient's symptoms. Nonetheless, successful control of painful symptoms of DPN can be achieved in many patients.

\section{REFERENCES}

1. Diabetes Control and Complications Trial Research Group. The effect of intensive treatment of diabetes on the development and progression of long-term complications in insulin-dependent diabetes mellitus. The Diabetes Control and Complications Trial Research Group. N Engl J Med 1993; 329: 977-986.

2. Diabetes Control and Complications Trial (DCCT) Research Group. Effect of intensive diabetes treatment on nerve conduction in the Diabetes Control and Complications Trial. Ann Neurol 1995; 38: 869-880.

3. UK Prospective Diabetes Study (UKPDS) Group. Intensive bloodglucose control with sulfonylureas or insulin compared with conventional treatment and risk of complications in patients with type 2 diabetes (UKPDS 33). Lancet 1998; 352: 837-853.

4. National Diabetes Data Group. Factors in development of diabetic neuropathy. Baseline analysis of neuropathy in feasibility phase of Diabetes Control and Complications Trial (DCCT). The DCCT Research Group. Diabetes 1988; 37: 476-481.

5. Zochodne D. Diabetic neuropathies: features and mechanisms. Brain Pathol 1999; 9: 369-391.

6. Vinik A. Diabetic neuropathy: pathogenesis and therapy. Am J Med 1999; 107: 17S-26S.

7. Sima A, Bril V, Nathaniel V, et al. Regeneration and Repair of
Myelinated Fibers in Sural Nerve Biopsy Specimens from Patients with Diabetic Neuropathy Treated with Sorbinil. N Engl J Med 1988; 319: 548-555.

8. Greene D, Stevens M, Feldman E. Glycemic control. In: Dyck P, Thomas P, eds. Diabetic Neuropathy. Philadelphia: WB Saunders, 1999: 297-316.

9. Low P, Nichander K, Scionti L. Role of hypoxia, oxidative stress, and excitatory neurotoxins in diabetic neuropathy. In: Dyck P, Thomas P, eds. Diabetic Neuropathy. Philadelphia: WB Saunders, 1999: 317-329.

10. Tomlinson D. Role of aldose reductase inhibitors in the treatment of diabetic polyneuropathy. In: Dyck P, Thomas P, eds. Diabetic Neuropathy. Philadelphia: WB Saunders, 1999: 330-340.

11. Brimijoin S. Axonal transport in diabetic neuropathy. In: Dyck P, Thomas P, eds. Diabetic Neuropathy. Philadelphia: WB Saunders, 1999: 341-352.

12. Brownlee M. Advanced glycation end products and diabetic peripheral neuropathy. In: Dyck P, Thomas P, eds. Diabetic Neuropathy. Philadelphia: WB Saunders, 1999: 353-358.

13. Cameron N, Cotter M. Role of linolenic acid in diabetic polyneuropathy. In: Dyck P, Thomas P, eds. Diabetic Neuropathy. Philadelphia: WB Saunders, 1999: 359-367.

14. Yasuda H, Kikkawa R. Role of antiprostaglandins in diabetic neuropathy. In: Dyck P, Thomas P, eds. Diabetic Neuropathy. Philadelphia: WB Saunders, 1999: 368-376.

15. Feldman E, Windebank A. Growth factors and peripheral neuropathy. In: Dyck P, Thomas P, eds. Diabetic Neuropathy. Philadelphia: WB Saunders, 1999: 377-386.

16. Melton L, Dyck P. Epidemiology. In: Dyck P, Thomas P, eds. Diabetic Neuropathy. Philadelphia: WB Saunders, 1999: 239254.

17. Tovi J, Svanborg E, Nilsson B, Engfeldt P. Diabetic neuropathy in elderly Type 2 diabetic patients: effects of insulin treatment. Acta Neurol Scand 1998; 98: 346-353.

18. Reichard P, Nilsson B, Rosenqvist U. The effect of long-term intensified insulin treatment on the development of microvascular complications of diabetes mellitus. N Engl J Med 1993; 329: 304-309.

19. Navarro X, Sutherland D, Kennedy W. Long-term effects of pancreatic transplantation on diabetic neuropathy. Ann Neurol 1997; 42: 727-736.

20. Pirart J. Diabetes mellitus and its degenerative complications: a prospective study of 4,400 patients observed between 1947 and 1973. Diabete Metab 1978; 1: 168-188, 252-263.

21. 1998 Clinical practice guidelines for the management of diabetes in Canada. Can Med Assoc J 1998; 159: 159.

22. ADA. Clinical practice recommendations 2001. Diabetes Care 2001; 24(Suppl 1): S33-S43.

23. Green A, Jaspan J. Treatment of diabetic neuropathy with inhibitors of the aldose reductase enzyme. J Diabet Complications 1990; 4: 138-144.

24. Greene D, Lattimer S, Sima A. Sorbitol, phospholipids, and sodiumpotassium-ATPase in the pathogenesis of diabetic complications. N Engl J Med 1987; 316: 599-606.

25. Sorbinil Retinopathy Trial Research Group. The sorbinil retinopathy trial: neuropathy results. Sorbinil Retinopathy Trial Research Group. Neurology 1993; 43: 1141-1149.

26. Sundkvist G, Armstrong F, Bradbury J, et al and United Kingdom/Scandinavian Ponalrestat Trial. Peripheral and autonomic nerve function in 259 diabetic patients with peripheral neuropathy treated with ponalrestat (an aldose reductase inhibitor) or placebo for 18 months. United Kingdom/Scandinavian Ponalrestat Trial. J Diabetes Complications 1992; 6: 123-130.

27. Ghahary A, Chakrabarti S, Sima A, Murphy L. Effect of insulin and statil on aldose reductase expression in diabetic rats. Diabetes 1991; 40: 1391-1396.

28. Willars G, Calcutt N, Tomlinson D. Reduced anterograde and retrograde accumulation of axonally transported phosphofructokinase in streptozotocin-diabetic rats: effects of insulin and the aldose reductase inhibitor 'Statil'. Diabetologia 1987; 30: 239-243. 
29. Faes T, Yff G, De Weerdt O, et al. Treatment of diabetic autonomic neuropathy with an aldose reductase inhibitor. J Neurol 1993; 240: $156-160$.

30. Sima A, Greene D, Brown M, et al and Tolrestat Study Group. Effect of hyperglycemia and the aldose reductase inhibitor tolrestat on sural nerve biochemistry and morphometry in advanced diabetic peripheral polyneuropathy. The Tolrestat Study Group. J Diabetes Complications 1993; 7: 157-169.

31. Asbury A, Porte D. Report and recommendations of the San Antonio conference on diabetic neuropathy. Ann Neurol 1988; 24: 99-104.

32. Cameron N, Cotter M, Dines K, et al. Aldose reductase inhibition, nerve perfusion, oxygenation and function in streptozotocindiabetic rats: dose-response considerations and independence from a myo-inositol mechanism. Diabetologia 1994; 37: 651-653.

33. Greene D, Arezzo J, Brown M and Zenarestat Study Group. Effect of aldose reductase inhibition on nerve conduction and morphometry in diabetic neuropathy. Zenarestat Study Group. Neurology 1999; 53: 580-591.

34. Uchida K, Kigoshi T, Nakano S, et al. Effect of 24 weeks of treatment with epalrestat, an aldose reductase inhibitor, on peripheral neuropathy in patients with non-insulin-dependent diabetes mellitus. Clin Ther 1995; 17: 460-466.

35. Hotta N, Sakamoto N, Shigeta Y, Kikkawa R, Goto Y. Clinical investigation of epalrestat, an aldose reductase inhibitor, on diabetic neuropathy in Japan: multicenter study. Diabetic Neuropathy Study Group in Japan. J Diabetes Complications 1996; 10: 168-172.

36. Di Giulio A, Lesma E, Gorio A. Diabetic neuropathy in the rat: 1. Alcar augments the reduced levels and axoplasmic transport of substance P. J Neurosci Res 1995; 40: 414-419.

37. Williamson J, Arrigoni-Martelli E. The roles of glucose-induced metabolic hypoxia and imbalances in carnitine metabolism in mediating diabetes-induced vascular dysfunction. Int J Clin Pharm Res 1992; 12: 247-252.

38. Gorio A, Di Giulio A, Tenconi B, et al. Peptide alterations in autonomic diabetic neuropathy prevented by acetyl-L-carnitine. Int J Clin Pharmacol Res 1992; 12: 225-230.

39. Gorio A, Di Giulio A, Donadoni L, et al. Early neurochemical changes in the autonomic neuropathy of the gut in experimental diabetes. Int J Clin Pharmacol Res 1992; 12: 217-224.

40. Bril V, Ellison R, Ngo M, et al and Roche Neuropathy Study Group. Electrophysiological monitoring in clinical trials. Roche Neuropathy Study Group. Muscle Nerve 1998; 21: 1368-1373.

41. Apfel S. Neurotrophic factors in peripheral neuropathies: therapeutic implications. Brain Pathol 1999; 9: 393-413.

42. Apfel S. Neurotrophic factors in the therapy of diabetic neuropathy. Am J Med 1999; 107: 34S-42S.

43. Rogers B. Development of recombinant human nerve growth factor (rhNGF) as a treatment for peripheral neuropathic disease. Neurotoxicology 1996; 17: 865-870.

44. Apfel S, Kessler J, Adornato B, et al. Recombinant human nerve growth factor in the treatment of diabetic polyneuropathy. NGF Study Group. Neurology 1998; 51: 695-702.

45. Freeman R. Human studies of recombinant human nerve growth factor and diabetic peripheral neuropathy. Eur Neurol 1999; 41: 20-26.

46. Apfel S, Schwartz S, Adornato B, et al. Efficacy and safety of recombinant human nerve growth factor in patients with diabetic polyneuropathy: a randomized controlled trial. JAMA2000; 284: 2215-2221.

47. Dyck P, Kratz K, Lehman K, et al. The Rochester Diabetic Neuropathy Study: design, criteria for types of neuropathy, selection bias, and reproducibility of neuropathic tests. Neurology 1991; 41: 799-807.

48. Bril V. NIS-LL: The primary measurement scale for clinical trial endpoints in diabetic peripheral neuropathy. Eur Neurol 1999; 41: 8-13.

49. O'Brien J, Carson G, Seo H, Hiraiwa M, Kishimoto Y. Identification of prosaposin as a neurotrophic factor. Proc Natl Acad Sci USA 1994; 91: 9593-9596.

50. Calcutt N, Campana W, Eskeland N, et al. Prosaposin gene expressin and the efficacy of a prosaposin-derived peptide in preventing structural and functional disorders of peripheral nerve in diabetic rats. J Neuropathol Exp Neurol 1999; 58: 628-636.

51. Ziegler D, Reljanovic M, Mehnert H, Gries F. Alpha-lipoic acid in the treatment of diabetic polyneuropathy in Germany: current evidence from clinical trials. Exp Clin Endocrinol Diabetes 1999; 107: 421-430.

52. Ziegler D, Hanefeld M, Ruhnau K, et al. Treatment of symptomatic diabetic peripheral neuropathy with the anti-oxidant alpha-lipoic acid. A 3-week multicentre randomized controlled trial (ALADIN Study). Diabetologia 1995; 38: 1425-1433.

53. Shiba T, Inoguchi $T$, Sportsman $J$, et al. Correlation of diacylglycerol level and protein kinase $\mathrm{C}$ activity in the rat retina to retinal circulation. Am J Physiol 1993; 265: E783-E793.

54. Cohen R. Dysfunction of vascular endothelium in diabetes mellitus. Circulation 1993; 87(suppl): V67-V76.

55. Cohen K, Lucibello F, Chomiak M. Lack of effect of clonidine and pentoxifylline in short-term therapy of diabetic peripheral neuropathy. Diabetes Care 1990; 13: 1074-1077.

56. Cohen S, Mathews T. Pentoxifylline in the treatment of distal diabetic neuropathy. Angiology 1991; 42: 741-746.

57. Hendriksen P, Oey $P$, Wieneke $G H$, Banga J, van Dam P. Antihypoxic treatment at an early stage of diabetic neuropathy: an electrophysiological study with sabeluzole. Acta Neurol Scand 1992; 86: 506-511.

58. Keen H, Payan J, Allawi J, et al. Treatment of diabetic neuropathy with gamma-linolenic acid. The Gamma-Linolenic Acid Multicenter Trial Group. Diabetes Care 1993; 16: 8-15.

59. Keen H, Payan J, Allawi J, et al. Treatment of diabetic neuropathy with gamma-linolenic acid. The Gamma-Linolenic Acid Multicenter Trial Group. Diabetes Care 1993; 16: 8-15.

60. Bril V, Ellison R, Ngo M, et al. Electrophysiological monitoring in clinical trials. Muscle Nerve 1998;21:1368-1373.

61. Harati Y. Diabetes and the nervous system. Endocrinol Metab Clin North Am 1996; 25: 325-359.

62. Perkins B, Olaleye D, Zinman B, Bril V. Simple screening tests for peripheral neuropathy in the diabetes clinic. Diabetes Care 2001; 24: $250-256$

61. Tanaka S, Wild DK, Seligman PJ, et al. The USprevalence of selfreported carpal tunnel syndrome. 1988 National Health interview survey data. Am J Pub Health 1994;84:1846-1848.

64. Pfeifer M, Ross D, Schrage J, et al. A highly successful and novel model for treatment of chronic painful diabetic peripheral neuropathy. Diabetes Care 1993; 16: 1103-1115.

65. Portenoy RK, Kanner RM, eds. Pain Management: Theory and Practice. Contemporary Neurology Series. vol 68. Philadelphia: Davis, FA, 1996.

66. McQuay H, Carroll D, Jadad A, Wiffen P, Moore A. Anticonvulsant drugs for management of pain: a systematic review. Br Med J 1995; 311: 1047-1052.

67. McQuay H, Tramer M, Nye B, et al. A systematic review of antidepressants in neuropathic pain. Pain 1996; 68: 217-227.

68. Harati Y, Gooch C, Swenson M, et al. Double-blind randomized trial of tramadol for the treatment of the pain of diabetic neuropathy. Neurology 1998; 50: 1842-1846.

69. Watson C, Watt-Watson J. Treatment of neuropathic pain: focus on antidepressants, opioids and gabapentin. Pain Res Manage 1999; 4: $168-178$.

70. Max M, Culnane M, Schafer S, et al. Amitriptylione relieves diabetic neuropathy pain in patients with normal or depressed mood. Neurology 1987; 37: 589-596.

71. Max M, Lynch S, Muir J, et al. Effects of desipramine, amitriptyline, and fluoxetine on pain in diabetic neuropathy. $\mathrm{N}$ Engl J Med 1992; 326: 1250-1256.

72. Kvinesdal B, Molin J, Froland A, Gram L. Imipramine treatment of painful diabetic neuropathy. JAMA1984; 251: 1727-1730.

73. Rull J, Quibrera R, Gonzalez-Millan H, Lozano Castaneda O. Symptomatic treatment of peripheral diabetic neuropathy with carbamazepine (Tegretol®): double-blind cross-over trial. Diabetologia 1969; 5: 215-218.

74. Gomez-Perez F, Rull J, Dies H, et al. Nortriptyline and fluphenazine in the symptomatic treatment of diabetic neuropathy. A doubleblind cross-over study. Pain 1985; 23: 395-400. 
75. Gomez-Perez F, Choza R, Rios J, et al. Nortriptyline-fluphenazine vs. carbamazepine in the symptomatic treatment of diabetic neuropathy. Arch Med Res 1996; 27: 525-529.

76. Wright J, Oki J, Graves L. Mexiletine in the symptomatic treatment of diabetic peripheral neuropathy. Ann Pharmacother 1997;31:29-34.

77. Kastrup J, Angelo H, Petersen P, Dejgard A, Hilsted J. Treatment of chronic painful diabetic neuropathy with intravenous lidocaine infusion. Br Med J (Clin Res Ed) 1986; 292: 173.

78. Backonja M, Beydoun A, Edwards K, et al and Gabapentin Diabetic Neuropathy Study Group. Gabapentin for the Symptomatic Treatment of Painful Neuropathy in Patients with Diabetes Mellitus. A Randomized Controlled Trail. JAMA 1998; 280: 1831-1836.

79. Morello C, Leckband S, Stoner C, Moorhouse D, Sahagian G. Randomized double-blind study comparing the efficacy of gabapentin with amitriptyline on diabetic peripheral neuropathy pain. Arch Intern Med 1999; 159: 1931-1937.

80. Galer B. Neuropathic pain of peripheral origin: advances in pharmacologic treatment. Neurology 1995; 45: S17-S25; discussion S35-36.

81. Vrethem M, Boivie J, Arnqvist $\mathrm{H}$, et al. A comparison of amitriptyline and maprotiline in the treatment of painful diabetic neuropathy in diabetics and nondiabetics. Clin J Pain 1997; 13: 313-323.

82. Young R, Clarke B. Pain relief in diabetic neuropathy: the effectiveness of imipramine and related drugs. Diabet Med 1985; 2: 363-366.

83. Davis J, Smith R. Painful peripheral diabetic neuropathy treated with venlafaxine $\mathrm{HCl}$ extended release capsules. Diabetes Care 1999; 22: 1909-1910.
84. Kiayias J, Vlachou E, Lakka-Papadodima E. Venlafaxine $\mathrm{HCl}$ in the treatment of painful peripheral diabetic neuropathy. Diabetes Care 2000; 23: 699.

85. Chakrabarti A, Samantaray S. Diabetic peripheral neuropathy: nerve conduction studies before, during and after carbamazepine therapy. Aust N Z J Med 1976; 6: 565-568.

86. Backonja M. Anticonvulsants (antineuropathics) for neuropathic pain syndromes. Clin J Pain 2000; 16: S67-S72.

87. Jarvis B, Coukell A. Mexiletine. A review of its therapeutic use in painful diabetic neuropathy. Drugs 1998; 56: 691-707.

88. Oskarsson P, Ljunggren J, Lins P and Mexiletine Study Group. Efficacy and safety of mexiletine in the treatment of painful diabetic neuropathy. The Mexiletine Study Group. Diabetes Care 1997; 20: 1594-1597.

89. Stracke H, Meyer U, Schumacher H, Federlin K. Mexiletine in the treatment of diabetic neuropathy. Diabetes Care 1992; 15: 15501555.

90. Tandan R, Lewis G, Krusinski P, Badger G, Fries T. Topical capsaicin in painful diabetic neuropathy. Controlled study with long-term follow-up. Diabetes Care 1992; 15: 8-14.

91. Capsaicin Study Group. Treatment of painful diabetic neuropathy with topical capsaicin. A multicenter, double-blind, vehiclecontrolled study. The Capsaicin Study Group. Arch Intern Med 1991; 151: 2225-2229.

92. Harati Y, Gooch C, Swenson M, et al. Maintenance of the long-term effectiveness of tramadol in treatment of the pain of diabetic neuropathy. J Diabetes Complications 2000; 14: 65-70.

93. Sindrup S, Jensen T. Pharmacologic treatment of pain in polyneuropathy. Neurology 2000; 55: 915-920. 